stimuli in fibromyalgia patients, but little is known about the mechanisms involved in processing painless stimuli.

This study compared brain responses to repeated, painless somatosensory and auditory stimuli in 15 female patients with fibromyalgia and 15 healthy control individuals. Participants underwent two sessions of electroencephalographic tests, with a 2 min rest period between sessions. Each session contained 40 paired stimuli, which could be either painless and tactile (pneumatic stimulator), or auditory. Mean event-related potentials (ERPs) in response to a pair of identical stimuli (S1 and S2) were calculated over the 40 paired trials of each sensory modality. In healthy controls, ERP amplitudes in response to the somatosensory and auditory S2 stimuli were markedly lower than those in response to $\mathrm{S} 1$ stimuli, which indicated (as expected) habituation in response to repeated stimulation. In 15 patients with fibromyalgia, similarly marked ERP amplitude reductions from S1 to S2 stimuli were seen for auditory stimuli, but the amplitude reductions from S1 to $\mathrm{S} 2$ in response to somatosensory stimuli were smaller than those of healthy controls.

The authors conclude that patients with fibromyalgia lack inhibitory control to repeated, painless somatosensory stimulation during stimulus coding and cognitive evaluation.

Original article Montoya P et al. (2006) Reduced brain habituation to somatosensory stimulation in patients with fibromyalgia. Arthritis Rheum 54: 1995-2003

\section{Factors associated with transition from primary to secondary Raynaud's phenomenon}

Patients with primary Raynaud's phenomenon have, at the time of diagnosis, no evidence of an underlying disease whereas those with secondary Raynaud's phenomenon have a wide variety of vascular and nonvascular diseases. It is unclear how often primary Raynaud's phenomenon progresses to secondary Raynaud's phenomenon, and whether prognostic factors for this transition, or for disease progression, exist.

This prospective, follow-up study assessed the rate of transition from primary to secondary Raynaud's phenomenon in 307 consecutive patients, 34 of whom had a confirmed diagnosis of secondary Raynaud's phenomenon. The initial prevalence of secondary Raynaud's phenomenon was $11 \%$. In the remaining patients, the annual rates of transition to suspected and confirmed secondary Raynaud's phenomenon were $2 \%$ and $1 \%$, respectively. An increased risk of transition to secondary Raynaud's phenomenon was associated with older age at onset, recent onset of Raynaud's phenomenon at enrolment, abnormal thoracic-outlet test results and antinuclear antibody titer $>1: 160$. Overall, patients with confirmed, secondary Raynaud's phenomenon had earlier onset, and a higher number, of pathologic findings.

The authors suggest that patients with primary Raynaud's phenomenon can be divided into three subsets: those with no sign of underlying disease at presentation or during long-term follow-up (idiopathic Raynaud's phenomenon), those with rapid onset and an increased number of pathologic findings (severe course of disease), and those with late onset of pathologic findings and a relatively benign disease course, apparently unrelated to other conditions.

Original article Hirschl M et al. (2006) Transition from primary Raynaud's phenomenon to secondary Raynaud's phenomenon identified by diagnosis of an associated disease: results of ten years of prospective surveillance. Arthritis Rheum 54: 1974-1981

\section{Clinical symptoms of osteoarthritis are associated with MRI structural findings}

The underlying cause of the knee pain experienced in osteoarthritis $(O A)$ is unclear. It is unlikely to be related to cartilage loss given the lack of pain fibres in this tissue, but the role of damage to other structures, such as the joint capsule, periosteum, menisci and synovium, has not been clarified.

To investigate whether structural abnormalities detected by MRI in the knees of patients with OA are associated with pain and stiffness, Kornaat and colleagues performed a prospective study in 205 patients with symptomatic OA in at least two joints (hands, spine, knees and/or hips). All patients underwent MRI of the knee, and the scans were evaluated using a comprehensive score for a range of structural abnormalities, by three clinicians blinded to patient data. No associations were found between pain and stiffness of the knee and focal or diffuse cartilaginous abnormalities, subchondral cysts, bone-marrow edema, subluxation of the meniscus, meniscal 